\title{
Niveles de alergenos de ácaros en el polvo de habitación en Cartagena, Colombia
}

\author{
Dilia Mercado, Leonardo Puerta, Luis Caraballo
}

\begin{abstract}
Resumen
Se investigó la cantidad de alergenos derivados de los ácaros Dermatophagoides pteronyssinus, Dermatophagoides faringe y Blomia tropicalis en el polvo de habitación de 20 casas de pacientes con asma alérgica inducida por ácaros domésticos. Las muestras se recolectaron en Cartagena, mensualmente durante un año, utilizando una aspiradora portátil modificada en el extremo succionador. Los niveles de alergenos Der $p$ 1 y Der $f 1$ se determinaron mediante un inmunoensayo con anticuerpos monoclonales. La cuantificación de los alergenos totales de $B$. tropicalis y $D$. pteronyssinus se realizó mediante un ensayo de inhibición del RAST. En el polvo de colchón, el nivel más alto del alergeno Der $p 1$ fue 109,49 ng/g de polvo, detectado en agosto. Este nivel fue superior al doble del nivel inferior $(50,3 \mathrm{ng} / \mathrm{g})$ detectado en noviembre. Der $f 1$ se encontró solamente en tres muestras de polvo de piso. Los niveles promedio más altos de los alergenos totales de $D$. pteronyssinus, en el polvo de colchón, se observaron entre mayo y agosto; los niveles más bajos se presentaron entre diciembre y abril. Los niveles promedio más altos de alergenos de $B$. tropicalis, en el polvo de colchón, se detectaron en junio, julio y agosto, el nivel promedio más bajo se presentó en septiembre. Los niveles de alergenos en el polvo del colchón se correlacionaron con los niveles en el polvo del piso, pero, fueron significativamente más altos que éstos $(p<0,0001)$. Los niveles de todos los alergenos presentaron una correlación significativa con la humedad absoluta. En este estudio, se describe que la variación durante el año de los niveles de alergenos de ácaros domésticos en un ambiente tropical es muy poca. Además, se describen niveles altos y constantes de los alergenos de $B$. tropicalis. Los pacientes alérgicos a los ácaros domésticos en Cartagena están expuestos, durante todo el año, a una carga alergénica importante.
\end{abstract}

\section{Summary}

Domestic mites Dermatophagoides pteronyssinus, Dermatophagoides faringe and Blomia tropicalis house dust allergen levels in Cartagena were investigated. Dust samples were collected monthly from the homes of twenty asthmatic patients in Cartagena having allergy to these mites, using a portable vacuum cleaner throughout a 12 -month period. The major allergens from $D$. pteronyssinus and $D$. faringe, Der $p 1$ and Der $f 1$ respectively, were determined by immunoassay using monoclonal antibodies. $D$. pteronyssinus and $B$. tropicalis total allergen levels were determined by RAST inhibition assay. The highest Der $p 1$ mean level in mattress samples was $109.49 \mathrm{ng} / \mathrm{g}$ dust detected in August and represented more than twice the lowest levels $(50.3 \mathrm{ng} / \mathrm{g})$ detected 
in November. Derf 1 was detected in only three floor-dust samples. The highest levels of $D$. pteronyssinus allergens in mattress samples were detected during the months of May, June, July and August and the lowest levels from December to April. The highest levels of $B$. tropicalis allergens in mattress samples were detected in June, July and August and the lowest levels during September. The allergen levels in mattress-dust which were correlated with floor-dust levels were significantly higher than the latter $(p<0.0001)$. There was a good correlation between $B$. tropicalis and $D$. pteronyssinus allergen levels and absolute humidity. This study describes the presentation of minimal variation, throughout the year, in allergen levels from domestic mites in a tropical environment, and that $B$. tropicalis allergen levels are high and constant. Patients allergic to domestic mites in Cartagena are exposed throughout the year to an important allergenic load.

Los alergenos de los ácaros domésticos son los principales inductores de alergias del sistema respiratorio (1). Debido al factor determinante de la humedad y la temperatura para el crecimiento de los ácaros, se ha investigado la relación entre el contenido de alergenos en el ambiente y los cambios climáticos provocados por las estaciones. La asociación entre estos factores se sugirió desde la demostración del papel alergénico de los ácaros presentes en el polvo de habitación $(2,3)$. En algunas regiones de los Estados Unidos, se ha demostrado que en determinadas estaciones del año los aumentos en la humedad relativa coinciden con el pico en el número de ácaros o de sus alergenos (4-6). Por otro lado, en el sur de Manchester, Inglaterra, no se hallaron variaciones estacionales en los niveles del alergeno mayor del ácaro D. pteronyssinus, Der $p 1$ (7).

Los reservorios de ácaros más importantes son: el colchón, la almohada, la sala de estar y las alfombras; en estos sitios es donde, también, se acumulan las mayores concentraciones de sus alergenos. La cuantificación de alergenos en el medio ambiente es importante para estimar el grado de exposición del paciente y para proponer y evaluar medidas de control ambiental. Entre las técnicas usadas para cuantificar los alergenos de ácaros en el ambiente, existe el ELISA que usa los anticuerpos monoclonales específicos a los alergenos más importantes y purificados (8) y la inhibición del RAST que usa una mezcla de sueros de individuos alérgicos con IgE específica a la especie de ácaro que se investiga. Mediante la inhibición del RAST, se detectan todos aquellos alergenos de un extracto que pueden ser identificados por la mezcla de sueros (9). Los extractos alérgenicos de los ácaros $D$. pteronyssinus y $D$. faringe contienen aproximadamente unos 30 alergenos, de los cuales $\operatorname{Der} p 1$ y $\operatorname{Der} f 1$ son los más importantes porque inducen alergia en más de $80 \%$ de la población asmática. Por ser tan representativos de los ácaros de donde proceden, su cuantificación en el ambiente se usa para estimar el grado de exposición a los alergenos de dichos ácaros $(8,10)$.

La fauna acarológica del polvo de habitación en Cartagena está constituida por $B$. tropicalis $(40,1 \%)$, D. pteronyssinus $(35,7 \%)$, Dermatophagoides faringe $(<1 \%)$ y el resto lo constituyen otras especies (9). Estos porcentajes para las especies del género Dermatophagoides, los más asociados con alergias del sistema respiratorio, son mucho más bajos que los descritos en otra regiones del mundo. Esto sugiere que los alergenos producidos por ácaros diferentes a los Dermatophagoides pueden tener un papel importante en la inducción de alergias en esta región. Tal es el caso de B. tropicalis, uno de los más frecuentes en el trópico y de gran alergenicidad $(9,11-14)$.

Cartagena tiene un clima cálido y húmedo durante todo el año, además, como otras ciudades tropicales, no presenta los cambios de estaciones observados en otras regiones. En esta ciudad, la prevalencia de asma es de $13 \%$ (15) y alrededor de $80 \%$ de los pacientes con asma tienen anticuerpos IgE contra los ácaros domésticos (16). Sin embargo, se desconoce cómo las condiciones climáticas afectan la carga alergénica en esta ciudad. 
El presente estudio se realizó con el propósito de establecer los niveles de alergenos totales de $B$. tropicalis y $D$. pteronyssinus, así como de los alergenos purificados $\operatorname{Der} p 1$ y $\operatorname{Der} f l$, para observar su relación con las condiciones climáticas en Cartagena. Este trabajo hace parte de un programa dirigido a determinar los factores de riesgo para el asma en un ambiente tropical.

\section{Materiales y métodos}

\section{Pacientes}

Se seleccionaron 20 pacientes con asma alérgica provocada por los ácaros del polvo casero, para analizar el contenido alergénico del polvo de habitación de sus casas. Todos los pacientes tuvieron pruebas cutáneas positivas (pápula $>3 \mathrm{~mm}$ ) a extractos glicerinados de $B$. tropicalis, 1:50 peso/vol [preparado en el Instituto de Investigaciones Inmunológicas (III)], $D$. pteronissinus y $D$. forinae (Greer Laboratories, Lenoir, NC). También presentaron títulos positivos de IgE específica mediante RAST a las diferentes especies de ácaros antes mencionados.

Las 20 casas analizadas están ubicadas en el área urbana de Cartagena, 19 de las cuales están construidas en cemento y una en madera. Todas con piso de cemento, ninguna con alfombras ni aire acondicionado y ninguna de las camas tenía recubrimiento con plásticos.

\section{Recoleción de las muestras de polvo}

Se recolectaron muestras de polvo de habitación durante un período de doce meses consecutivos. Utilizando una aspiradora portátil modificada en el extremo succionador, se aspiró el polvo del colchón y del piso alrededor de la cama del paciente en una relación de $1 \mathrm{~m}^{2}$ de superficie por 2 mio. El contenido del polvo se retuvo en un papel de filtro y se guardó a $-20^{\circ} \mathrm{C}$ hasta la extracción de los alergenos.

La temperatura, la humedad relativa ambiental y las lluvias durante el período de recolección se obtuvieron de los datos del HIMAT (17). La humedad absoluta se calculó a partir de los datos de humedad relativa y temperatura usando un nomograma.

\section{Extración de alergenos del polvo de habitación}

Para obtener los alergenos a partir de las muestras de polvo, se pasaron por un tamiz para retirarle el material grueso, el polvo fino se sometió a un proceso de extracción en solución amortiguada de fosfato salina/glicerina, $\mathrm{pH} 7,4$ (1:60 peso/vol) durante 20 horas a $4^{\circ} \mathrm{C}$ y se centrifugaron. El sobrenadante que contenía los alergenos se guardó a $-20^{\circ} \mathrm{C}$ hasta el día del análisis.

\section{Extractos alergénicos}

Para la construcción de la curva de calibración usada en la prueba de inhibición del RAST, se utilizó un extracto alergénico estandarizado de D. pteronyssinus de 10.000 unidades de alergia (UA)/mL (Greer Laboratories, Lenoir, NC) y un extracto alergénico de $B$. tropicalis preparado a partir de los ácaros, cultivados en el III, extraidos en solución amortiguado de bicarbonato de amonio, 0,1 M (18). A este extracto se le asignó una concentración de $25.000 \mathrm{UA} / \mathrm{mL}$.

\section{Anticuerpos monoclonales}

Para el ensayo de ELISA, se obtuvieron de la Universidad de Virginia, EUA, los siguientes anticuerpos monoclonales: $5 \mathrm{H} 8$ (isotipo $\operatorname{lgG} 2 \mathrm{a}$ ), específico para Der $p$ 1, $6 A 8$ (isotipo IgG1), específico para Derf 1 y $4 \mathrm{C} 1$ (isotipo IgG1), dirigido contra epítopes comunes de Der plyDerfl.

\section{Ensayo de inhibición del RAST}

Para la cuantificación de los alergenos totales de $B$. tropicalis y de $D$. pteronyssinus, se realizó la prueba de inhibición del RAST siguiendo un método descrito previamente (18) que, brevemente, es el siguiente: los pozos de placas de microtitulación Inmulon 4 (Dynatech Laboratories, Alexandria, VA) se incubaron con $1 \mu \mathrm{g}$ de proteína del extracto de $B$. tropicalis o $1 \mu \mathrm{g}$ del extracto de $D$. pteronyssinus por 16 horas, se lavaron con solución amortiguada de fosfato salino-tween 20 (PBS-T) y se incubaron por 16 horas con $100 \mu \mathrm{L}$ de una mezcla de $50 \mu \mathrm{L}$ del extracto de polvo de habitación con $50 \mu \mathrm{L}$ de un pool de sueros de pacientes alérgicos a $B$. 
tropicalis y $D$. pteronyssinus. Después de varios lavados con PBS-T, se incubaron con $100 \mu \mathrm{L}$ de i25I-IgE que contenía $25.000 \mathrm{cpm}$ (Kallestad, Chaska, MN) durante 18 horas. La radioactividad se determinó en un contador de radiación gamma, previo lavado de los pozos con PBS-T. El porcentaje de inhibición obtenido se extrapoló en una curva estándar construida con concentraciones conocidas de alergenos de $B$. tropicalis o D. pteronyssinus.

\section{ELISA para cuantificar Der $p 1$ y Derf 1}

Los niveles de los alergenos mayores Der $p 1 y$ Der $f 1$ se determinaron por medio de un inmunoensayo de doble sitio con anticuerpo monoclonales (8). Se incubaron pozos de placas de microtitulación con $1 \mu \mathrm{g}$ de los monoclonales $5 \mathrm{H} 8$ específico para Der $p$ 1, o 6 A8 específico para $\operatorname{Der} f 1$, en solución amortiguada de bicarbonato $0,1 \mathrm{M}$ por 16 horas; se lavaron dos veces con PBS-T y se incubaron por $30 \mathrm{~min}$ con PBS-T/BSA al $1 \%$. Después de dos lavados, se incubaron con $100 \mu \mathrm{L}$ de extracto de polvo casero por 1 hora; se lavaron cinco veces con PBS-T y se incubaron con el monoclonal 4C1 dirigido a Der $p 1$ y $\operatorname{Der} f 1$, marcado con biotina. Terminada esta incubación, se lavaron como se indicó anteriormente y se incubaron por 30 minutos con $100 \mu \mathrm{L}$ de peroxidasa marcada con estreptovidina. Después de lavar con PBS-T se agregaron $100 \mu \mathrm{L}$ de ABTS (2,2'-azino-di-(3-ácido sulfonic-ethilbenzotiazolina) $1 \mathrm{mM}$ con peróxido de hidrógeno y a los 5 min se detuvo la reacción con azida de sodio $0,2 \mathrm{M}$. Se leyó la absorbancia a $414 \mathrm{~nm}$ en un lector de ELISA. Los valores de absorbancia se extrapolaron en una curva control construida con estándares de Der $p$ la Der $f$ 1. Los resultados se expresaron en $\mathrm{ng}$ de cada alergeno por $\mathrm{g}$ de polvo (ng/g).

\section{Análisis estadístico}

A partir de los niveles de alergenos mensuales de cada una de las 20 casas, se obtuvo una media geométrica de las concentraciones alérgenicas en cada mes. Las correlaciones se calcularon con los valores promedios de cada mes y los datos climatológicos mensuales informados por el HIMAT usando el coeficiente de correlación por rangos de Spearman.

\section{Resultados}

\section{Niveles de alergenos de $B$. tropicalis}

En todas las muestras de polvo de colchón se detectaron alergenos de $B$. tropicalis, en niveles entre $152 \mathrm{UA} / \mathrm{g}$ de polvo (15\% de inhibición del RAST) y $2.003 \mathrm{UA} / \mathrm{g}$ de polvo ( $80 \%$ de inhibición del RAST). En $30 \%$ de las muestras de polvo de colchón y en $4,3 \%$ de las muestras de polvo de piso se obtuvo una inhibición superior a $50 \%$. Esto indica un alto contenido de alergenos de $B$. tropicalis en las casas. Los niveles más altos de alergenos de $B$. tropicalis en las muestras de colchón se detectaron durante junio, julio y agosto, observándose incrementos de 1,44; 1,75 y 1,44 veces los niveles detectados en mayo, respectivamente. Sólo la diferencia entre mayo y julio fue estadísticamente significativa. El nivel promedio más bajo fue $287,3 \mathrm{UA} / \mathrm{g}$ en septiembre y el nivel promedio más alto fue $608,9 \mathrm{UA} / \mathrm{g}$ en julio. Este valor equivale a 2,1 veces el valor más bajo $(p=0,004)$. Los niveles más bajos se detectaron entre septiembre de 1992 y mayo de 1993 (figura 1). Con excepción de septiembre y octubre, en ese período, se presentaron bajos valores de humedad absoluta y de lluvias.

Los niveles de alergenos fueron significantemente más altos en las muestras de polvo de colchón que en las muestras de piso $(p=0,0001)$. Los niveles de alergenos en las muestras de polvo de colchón presentaron una correlación significativa con las del piso $(r=0,65, p=0,02)$.

\section{Niveles de alergenos de $D$. pteroyssinus}

En todas las muestras de polvo de colchón, se detectaron alergenos de $D$. pteronyssinus. EI nivel más alto fue $8.994 \mathrm{UA} / \mathrm{g}$ de polvo lo cual corresponde a un $80 \%$ de inhibición del RAST, el nivel más bajo fue $150 \mathrm{AU} / \mathrm{g}$ ( $5 \%$ de inhibición). En $13 \%$ de las muestras de polvo del colchón, se obtuvo una inhibición igual o superior al $50 \%$ de inhibición del RAST. El promedio más alto en los niveles de alergenos de $D$. pteronyssinus, en el polvo de colchón, fue $678,7 \mathrm{UA} / \mathrm{g}$ detectado en julio. Este valor representa un incremento de 1,6 veces sobre el valor más bajo de $420,93 \mathrm{AU} / \mathrm{g}$ detectado en abril. Los valores más altos en los niveles de alergenos se encontraron durante 


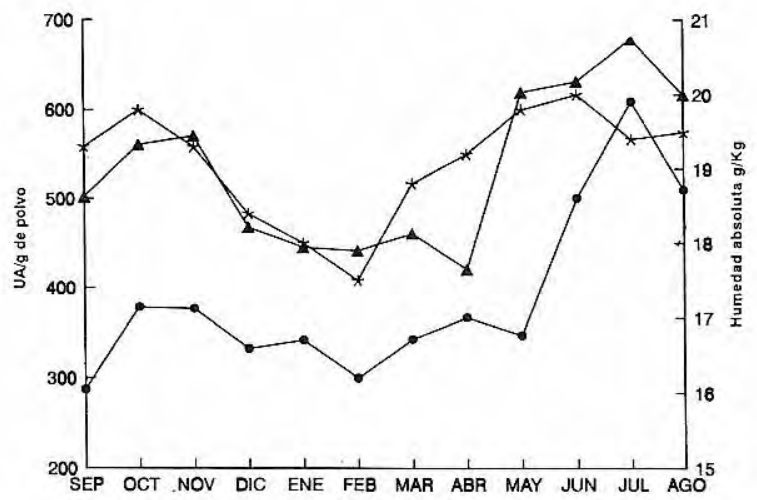

Figura 1. Niveles de alergenos totales, UA/g de polvo, de Blomia tropicalis (...) y Dermatophagoides pteronyssinus (..) en muestras de polvo de colchón y humedad absoluta, $\mathrm{g} / \mathrm{kg}\left(^{\star \star \star}\right)$. Los niveles más altos de alergenos se presentaron durante los meses de mayor humedad.

mayo, junio y julio. Los niveles de alergenos de $D$. pteronyssinus fueron significativamente más altos en el polvo del colchón que en el polvo del piso $(\mathrm{p}=0,0001)$.

\section{Niveles de Der $p 1$}

El valor promedio más alto en los niveles de Der $p 1$ en las muestras de polvo de colchón, 109,49 $\mathrm{ng} / \mathrm{g}$ se detectó en agosto y representa un incremento de 1,8 veces el nivel de $60,7 \mathrm{ng} / \mathrm{g}$ obtenido durante el mes inmediatamente anterior y un incremento de 2,2 veces del nivel más bajo obtenido durante noviembre. Las cantidades de Der $p 1$ en el polvo del piso se encontraron entre un rango de $30,23 \mathrm{ng} / \mathrm{g}$ (enero y diciembre) y $45,96 \mathrm{ng} / \mathrm{g}$ (junio); estos valores fueron significativamente más bajos que los detectados en el polvo de colchón (figura 2). Las cantidades de alergenos, tanto en el polvo del colchón como en el polvo del piso, fueron inferiores a los $2,0 \mu \mathrm{g}$ de Der $p \mathrm{t} / \mathrm{g}$ de polvo, sugeridos como nivel mínimo para producir sensibilización (19); por tanto, se pueden considerar bajos.

\section{Niveles de Derf 1}

Niveles de Der $f 1$ en colchón sólo se detectaron en tres casas en un rango entre $90 \mathrm{ng} / \mathrm{g}$ a1.600 $\mathrm{ng} / \mathrm{g}$ de polvo. El nivel más alto se detectó en la casa 5 en marzo y en las casas 10 y 16 en

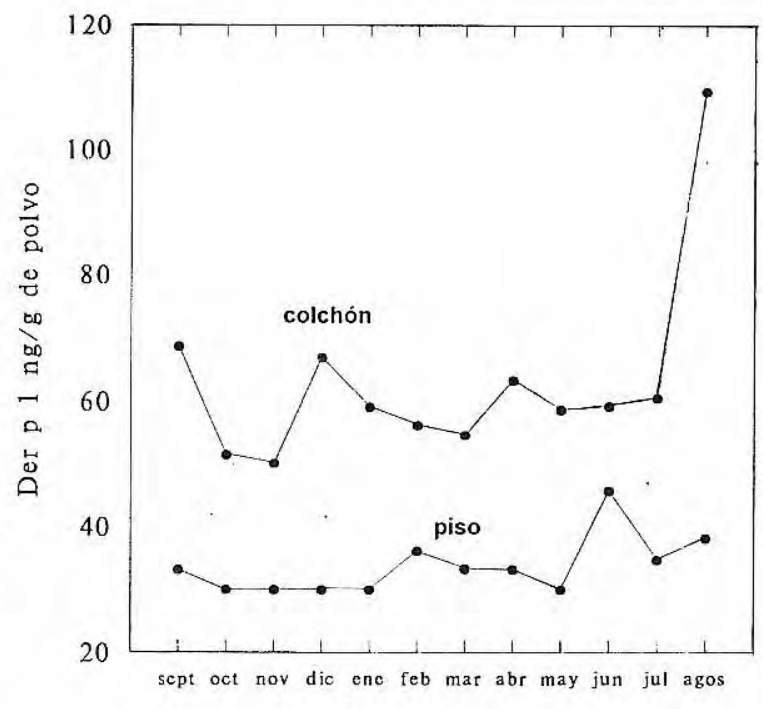

Figura 2. Niveles del alergeno Der $p 1$ en muestras de polvo del colchón y del piso.

febrero. Los niveles de Der $f 1$ en el polvo del piso se encontraron en un rango de $550 \mathrm{ng} / \mathrm{g}$ a $1,087 \mathrm{ng} / \mathrm{g}$ en la casa 10 en junio y julio y en la casa 16 en enero y febrero.

\section{Correlación entre los niveles de alergenos y la humedad}

Se halló correlación significativa entre la humedad absoluta y los niveles de alergenos de B. tropicalis en el colchón $(r=0,6, p=0,04)$ y en el piso $(r=0,68, p=0,02)$. También se halló una correlación significativa entre los niveles de alergenos de $D$. pteronyssinus en colchón y la humedad absoluta $(r=0,8, p=0,007)$. Sin embargo, no se encontró correlación entre los niveles de alergenos y la humedad relativa, ni con la cantidad de lluvias.

\section{Discusión}

Este estudio demuestra que los niveles de alergenos de $B$. tropicalis y $D$. pteronyssinus tienen mínimas variaciones durante el año en un área donde no se presentan las cuatro estaciones. En ningún mes se obtuvo un aumento mayor a dos veces el nivel del mes anterior. Sin embargo, se observaron dos picos en los niveles de alergenos de $D$. pteronyssinus: uno en de mayo a julio y el otro en octubre y noviembre. Los niveles de alergenos de $B$. tropicalis 
presentaron un pico entre junio y agosto. Estos picos ocurrieron durante los meses con valores más altos de humedad absoluta (figura 1). El aumento en los niveles de alergenos durante el segundo semestre del año, probablemente, se debe al efecto de mayores temperaturas, humedad y lluvias, siendo estas condiciones muy favorables para la proliferación de los ácaros, especialmente $B$. tropicalis y $D$. pteronyssinus. Aunque el alergeno $\operatorname{Der} f 1$ fue escasamente detectado y Der $p 1$ en cantidades menores a $2 \mathrm{ug} / \mathrm{g}$ de polvo, lo que indica un nivel bajo, las cantidades de alergenos totales de $D$. pteronyssinus y $B$. tropicalis alcanzaron niveles altos, como lo indica el porcentaje alto de inhibición en el ensayo de inhibición del RAST.

En dos casas se observó un aumento significativo (más de 10 veces el valor mínimo) en los niveles de alergenos de $D$. pteronyssinus lo cual puede reflejar el efecto de un microclima o microambiente particular en esas casas. El tipo de piso, la presencia o ausencia de alfombras, la tapicería de los muebles, como también el microclima de las casas, el clima exterior, las actividades domésticas y el tipo de construción de las casas son factores que influyen en el microambiente de los ácaros del polvo casero $(20,21)$. Estos factores deben tenerse en cuenta para explicar la recurrencia de síntomas de asma en un determinado paciente así como también para efectos de recomendar medidas de control ambiental.

En 1974, Cookson y Makoni (22) demostraron que los síntomas de asma en pacientes alérgicos a $D$. pteronyssinus en Salisbury, Rodesia, ciudad localizada en Africa tropical y con estaciones, se incrementaban en enero a marzo; este período corresponde, en ese sitio, a la estación caliente en la cual la humedad relativa y la temperatura tienen valores de 70 a $80 \%$ y 22 a $24^{\circ} \mathrm{C}$, respectivamente. Cartagena es una ciudad donde el período de lluvias se presenta de abril a noviembre y las temperaturas $y$ humedades son altas durante todo el año. En esta ciudad, no se ha establecido si se presenta un aumento en la sintomatología de asma en determinadas épocas del año. Sin embargo, los niveles altos y constantes de alergenos de ácaros durante todo el año, demostrada en este estudio, probablemente es uno de los factores que explican la alta prevalencia de enfermedades alérgicas respiratorias en esta ciudad (15, 23). En efecto, datos obtenidos de estudios en la sala de urgencias y en el Servicio de Otorrinolaringología del Hospital Universitario de Cartagena indican que la mayoría de los pacientes con alergia de tipo respiratorio tienen sensibilización a los ácaros $(24,25)$.

En un estudio previo, se halló que $D$. pteronyssinus y $D$. faringe constituyen menos de $38 \%$ de la fauna de ácaros presentes en el polvo de habitación de Cartagena (9). Esto podría explicar los bajos niveles de Der $p 1$ y Der $f 1$ encontrados en este estudio. Estos resultados confirman el hallazgo previo de que los niveles de $\operatorname{Der} p 1$ y $\operatorname{Der} f 1$ son bajos en el polvo de habitación recolectado en las casas de pacientes asmáticos que viven en el área urbana de Cartagena (9). Además, muestran que estos niveles permanecen bajos durante todo el año. En climas tropicales, parece ser que no existe mucha fluctuación en los niveles de alergenos, a diferencia de otras regiones como en Virginia y Ohio donde se han informado diferencias del orden de 20 a 100 veces en los niveles de alergenos o en el número de ácaros $(4,5)$.

A pesar de los bajos niveles de Der $p$ 1, la cuantificación de los alergenos totales de $D$. pteronyssinus realizada por inhibición del RAST alcanzó valores altos. En $13 \%$ de las muestras de polvo en el colchón, se obtuvo una inhibición igual o superior a $50 \%$. Este resultado se debe a que el ensayo de inhibición del RAST cuantifica, además del alergeno $\operatorname{Der} p 1$, a los otros alergenos específicos de $D$. pteronyssinus y a los alergenos de reactividad cruzada que comparten determinantes alergénicos con alergenos de otras especies de ácaros, probablemente, presentes en las muestras analizadas. Por ejemplo, Pyroglyphas amiicanus hallado en las muestras de polvo casero en esta ciudad (9), probablemente comparte determinantes alergénicos con $D$. pteronyssinus debido a la similitud filogenética con este ácaro. Además, $B$. tropicalis, el ácaro predominante en esta ciudad y Chortoglyphns arcuatus, también 
común en Cartagena, tienen algunos determinantes alergénicos compartidos con $D$. pteronyssinus $(18,26,27)$. Lo anterior sugiere que, en regiones con una fauna acarológica variada, el contenido alergénico del polvo de habitación se estima mejor con el uso de la prueba de inhibición del RAST o con la cuantificación de varios alergenos mayores de las especies de ácaros más numerosas en dicha región.

El uso de anticuerpos monoclonales para la cuantificación de los alergeno mayores, $\operatorname{Der} p 1$ y $\operatorname{Der} f 1$, son una herramienta importante para determinar la exposición a los alergenos de las especies Dermatophagoides $(8,10)$. Sin embargo, los anticuerpos monoclonales sólo permiten la cuantificación de alergenos específicos de especie, omitiendo otros alergenos presentes. El ensayo de inhibición del RAST es útil en la determinación de la mayoría de los determinantes alergénicos, incluyendo aquellos compartidos por varias especies de ácaros (26). De esta forma, se obtiene una idea más real de la exposición de alergenos en ambientes tropicales, donde otras especies de ácaros son comunes.

\section{Agradecimientos}

Este trabajo fue financiado por COLCIENCIAS y la Universidad de Cartagena.

\section{Referencias}

1. Moreno L, Caraballo L, Puerta L. Importancia médica de los ácaros domésticos. Biomédica 1995; 15:93-103.

2. Voorhorst R, Spieksman FT, Varekarnp H, eds. House dust atopy and the house dust mite. Leiden: Stafleu's Scientific Publishing Co.; 1969.

3. Voorhorst R, Spieksma FT, Varekamp H, Leupen MJ, Lyklema AW. The house dust mite (Dermatophagoides pteronissinus) and the allergens it produce: identity with the house dust allergens. J Allergy 1967;39:325.

4. Arlian LG, Bernstein IL, Gallagher JS. The prevalence of house dust mites, Dermatophagoides ssp., an associated enviromental conditions in homes in Ohio. J Allergy Clin Immunol 1982;69:527-32.
5. Platts-Milis TA, Hayden ML, Chapman MD, Wilkins SR. Seasonal variations in dust mite andgrass-pollen allergens in dust from the houses of patients with asthma. J Allergy Clin Immunol 1987;79:781-91.

6. Lintner T, Brame K. The effects of season, climate and air conditioning on the prevalence of Dermatophagoides mite allergens in household dust. J Allergy Clin Immunol 1993;91 862-7.

7. Karla S, Crank P, Hepworth J, Pickering CA, Woodcock A. Absence of seasonal variation in concentration of the house dust mite allergens $\operatorname{Der} p 1$ in South Manchester homes. Thorax 1992;47:928-31.

8. Luczynska CM, Atuda LK, Platts-Mills TAE, Miller JD, López M, Chapman MD. A two-site monoclonal antibody ELISA for the quantitation of the major Dermathophagoides spp. allergens, Der $p 1$ and Der $f$ 1. J Immunol Methods 1989;118:227-35.

9. Fernández-Caldas $E$, Puerta $L$, Mercado D, Lockey R, Caraballo L. Mite fauna, Der $p$ I, 16 Der $f$ and Blomia tropicalis allergen levels in a tropical city. Clin Exp Allergy 1993;23:292-7.

10. Platts-Mills T, Chapman M. Dust mites: immunology, allergenic disease and enviromental control. J Allergy Clin Immunol 1987;80:755-73.

11. Hurtado I, Parini M. House dust mite in Caracas, Venezuela. Ann Allergy 1987;59:128-30.

12. Vargas $\mathbf{M}$, Mairena $\mathbf{H}$. House dust from the metropolitan area of San José, Costa Rica. Internat J Acarol 1991;88:595-603.

13. Gabriel M, Cunington AM, Allan WG, Pickening CA, Wraith DG. Mite allergy in Hong Kong. Clin Allergy 1982;12:157-71.

14. Caraballo L, Puerta L, Martínez B, Moreno L. Identification of allergens from the mite Blomia tropicalis. Clin Exp Allergy 1994;24:1056-60.

15. Caraballo L, Cadavid A, Mendoza J. Prevalence of asthma in Cartagena, Colombia Ann Allergy 1992;68: 525-9.

16. Puerta L, Fernández-Caldas E, Lockey RF, Caraballo L. Mite allergy in the tropics: sensitization to six domestic mite species in Cartagena, Colombia. J Invest Allergol Clin Immunol 1993;3:198-204.

17. Calendario metereológico. Instituto Colombiano de Hidrología y Metereología (HIMAT) PE-AM, Santa Fe de Bogotá 1992-1993.

18. Puerta L, Fernández-Caldas E, Caraballo L. Alergenicidad cruzada entre los ácaros domésticos. Acta Med Colomb 1993;18:298-303.

19. Sporick R, Holgate ST, Platts-Mills TAE, Cogswels J. Exposure to house dust mite allergens (per $p$ 1) and the development of asthma in childhood. N Engl J Med 1990;323:502-7.

20. Hart BJ, Whitehead L. Ecology of house dust mites in Oxfordshire. Clin Exp Allergy 1990;20:203-9. 
21. Harving H, Korsgaard J, Dahl R. House-dust mites and associated environmental conditions in Danish homes. Allergy 1993;48:106-9.

22. Cookson JB, Makoni G. Seasonal asthma and the house-dust mite in tropical Africa. Clinical Allergy 1975;5:375-80.

23. Vergara C, Caraballo L. Algunas características epidemiológicas del asma en una región tropical. Rev Colomb Neumol 1995;7:187-91.

24. Puerta L, Mercado R, Martínez B, FernándezCaldas E, Arlian LG, Lockey R, Caraballo L. Emergency room: asthma and domestic mites in Cartagena,
Colombia (abstract) J Allergy Clin Immunol 1993;91: 354.

25. Marrugo J, Pérez L, Puerta L, Jiménez A, Moreno L, Martínez B, Caraballo L. The prevalence of allergen specific IgE response in patients with rhinitis (Abstract). J Allergy Clin Immunol 1993;91:199.

26. Puerta L, Fernández-Caldas E, Caraballo L. Lockey R. Sensitization to Chortoglyphns arcuatus and Aleuroglyphas ovatus in Dermatophagoides spp. allergic individuals. Clin Exp Allergy 1993;23:117-23.

27. Arlian LG, Vyszenski-Mohers DL, FernándezCaldas E. Allergenicity of the mite Blomia tropicalis. J Allergy Clin Immunol 193;91:1042-50. 